\title{
Genome-wide analysis suggests high level of microsynteny and purifying selection affect the evolution of EIN3/EIL family in Rosaceae (\#15747)
}

First revision

Please read the Important notes below, the Review guidance on page 2 and our Standout reviewing tips on page 3 . When ready submit online. The manuscript starts on page 4.

Important notes

\section{Editor}

Marion Röder

Files

1 Tracked changes manuscript(s)

1 Rebuttal letter(s)

7 Figure file(s)

2 Table file(s)

2 Other file(s)

Please visit the overview page to download and review the files not included in this review PDF.

Declarations

No notable declarations are present 
Please read in full before you begin

\section{How to review}

When ready submit your review online. The review form is divided into 5 sections. Please consider these when composing your review:

\section{BASIC REPORTING}

2. EXPERIMENTAL DESIGN

3. VALIDITY OF THE FINDINGS

4. General comments

5. Confidential notes to the editor

You can also annotate this PDF and upload it as part of your review

To finish, enter your editorial recommendation (accept, revise or reject) and submit.

\section{BASIC REPORTING}

Clear, unambiguous, professional English language used throughout.

Intro \& background to show context. Literature well referenced $\&$ relevant.

Structure conforms to PeerJ standards, discipline norm, or improved for clarity.

Figures are relevant, high quality, well labelled \& described.

Raw data supplied (see PeerJ policy).

\section{EXPERIMENTAL DESIGN}

Original primary research within Scope of the journal.

Research question well defined, relevant $\&$ meaningful. It is stated how the research fills an identified knowledge gap.

Rigorous investigation performed to a high technical \& ethical standard.

Methods described with sufficient detail \& information to replicate.

\section{VALIDITY OF THE FINDINGS}

Impact and novelty not assessed. Negative/inconclusive results accepted. Meaningful replication encouraged where rationale $\&$ benefit to literature is clearly stated.

Data is robust, statistically sound, $\&$ controlled.
Conclusions are well stated, linked to original research question \& limited to supporting results.

Speculation is welcome, but should be identified as such.

The above is the editorial criteria summary. To view in full visit https://peerj.com/about/editorialcriteria/ 
The best reviewers use these techniques

Tip

\author{
Support criticisms with \\ evidence from the text or from \\ other sources
}

\section{Give specific suggestions on how to improve the manuscript}

\section{Comment on language and grammar issues}

Organize by importance of the issues, and number your points

\section{Example}

Smith et al (J of Methodology, 2005, V3, pp 123) have shown that the analysis you use in Lines 241-250 is not the most appropriate for this situation. Please explain why you used this method.

Your introduction needs more detail. I suggest that you improve the description at lines 57- 86 to provide more justification for your study (specifically, you should expand upon the knowledge gap being filled).

The English language should be improved to ensure that your international audience can clearly understand your text. I suggest that you have a native English speaking colleague review your manuscript. Some examples where the language could be improved include lines 23, 77, 121, 128 - the current phrasing makes comprehension difficult.

1. Your most important issue

2. The next most important item

3. ...

4. The least important points

Line 56: Note that experimental data on sprawling animals needs to be updated. Line 66: Please consider exchanging "modern" with "cursorial".

I thank you for providing the raw data, however your supplemental files need more descriptive metadata identifiers to be useful to future readers. Although your results are compelling, the data analysis should be improved in the following ways: $A A, B B, C C$

I commend the authors for their extensive data set, compiled over many years of detailed fieldwork. In addition, the manuscript is clearly written in professional, unambiguous language. If there is a weakness, it is in the statistical analysis (as I have noted above) which should be improved upon before Acceptance.
Comment on strengths (as well as weaknesses) of the manuscript




\title{
Genome-wide analysis suggests high level of microsynteny and purifying selection affect the evolution of EIN3/EIL family in Rosaceae
}

\author{
Yunpeng Cao ${ }^{\text {Corresp.., }}{ }^{1}$, Yahui Han ${ }^{2}$, Dandan Meng ${ }^{1}$, Dahui Li ${ }^{1}$, Qing Jin ${ }^{1}$, Yi Lin ${ }^{1}$, Yongping Cai ${ }^{1}$ \\ ${ }^{1}$ college of Life Sciences, Anhui Agricultural University, Hefei, China \\ 2 State Key Laboratory of Tea Plant Biology and Utilization, Anhui Agricultural University, Hefei, China \\ Corresponding Author: Yunpeng Cao \\ Email address: 499981806@qq.com
}

The ethylene-insensitive3/ethylene-insensitive3-like (EIN3/EIL) protein ${ }_{1}$ are a type of nuclear-localized proteins with DNA-binding activity in plants. Although the EIN3/EIL gene family has been studied in several plant species, little is known about comprehensive study of the EIN3/EIL gene family in Rosaceae. In this study, 10, 5, 4, and 5 EIN3/EIL genes were identified in the genomes of pear (Pyrus bretschneideri), mei (Prunus mume), peach (Prunus persica) and strawberry (Fragaria vesca), respectively. Twenty-eight chromosomal segments of EIL/EIN3 gene family were found in four Rosaceae species, and these segments could form seven orthologous or paralogous groups based on interspecies or intraspecies gene colinearity (microsynteny) analysis. Moreover, the highly conserved regions of microsynteny were found in four Rosaceae species. Subsequent it was found that both whole genome duplication and tandem duplication events significantly contributed to the EIL/EIN3 gene family expansion. Gene expression analysis of the EIL/EIN3 genes in pear revealed subfunctionalization for several PbEIL genes derived from whole genome duplication. Noteworthy, according to environmental selection pressure analysis, the strong purifying selection should dominate the maintenance of the EIL/EIN3 gene family in four Rosaceae species. These results provided useful information of Rosaceae EIL/EIN3 genes, as well as insights into the evolution of this gene family in four Rosaceae species. Furthermore, high level of microsynteny in the four Rosaceae plants suggested that a large-scale genome duplication event in EIL/EIN3 gene family was predated to speciation. 
1 Genome-wide analysis suggests high level of microsynteny and purifying selection affect the

2 evolution of $E I N 3 / E I L$ family in Rosaceae

3

4 Yunpeng Cao ${ }^{1}$, Yahui Han ${ }^{2}$, Dandan Meng ${ }^{1}$, Dahui Li ${ }^{1}$, Qing Jin ${ }^{1}$, Yi Lin ${ }^{1}$ and Yongping Cai ${ }^{1 *}$

$5 \quad$ Affiliation

61 School of Life Sciences, Anhui Agricultural University, Hefei 230036, China

72 State Key Laboratory of Tea Plant Biology and Utilization, Anhui Agricultural University, Hefei 230036, China

$8 \quad$ * Correspondence: ypcaiah@163.com; Tel.: +86 551 65786137; Fax: +86 55165786340

9 


\section{Abstract}

25 The ethylene-insensitive3/ethylene-insensitive3-like (EIN3/EIL) protein $_{2}$ are a type of nuclear-localized proteins with DNA-binding activity in plants. Although the EIN3/EIL gene family has been studied in several plant species, little is known about comprehensive study of the EIN3/EIL gene family in Rosaceae. In this study, 10, 5, 4, and 5 EIN3/EIL genes were identified in the genomes of pear (Pyrus bretschneideri), mei (Prunus mume), peach (Prunus persica) and strawberry (Fragaria vesca), respectively. Twenty-eight chromosomal segments of EIL/EIN3 gene family were found in four Rosaceae species, and these segments could form seven orthologous or paralogous groups based on interspecies or intraspecies gene colinearity (microsynteny) analysis. Moreover, the highly conserved regions of microsynteny were found in four Rosaceae species. Subsequent it was found that both whole genome duplication and tandem duplication events significantly contributed to the EIL/EIN3 gene family expansion. Gene expression analysis of the EIL/EIN3 genes in pear revealed subfunctionalization for several $P b E I L$ genes derived from whole genome duplication.

Noteworthy, according to environmental selection pressure analysis, the strong purifying selection should dominate the maintenance of the EIL/EIN3 gene family in four Rosaceae species. These results provided useful information of Rosaceae EIL/EIN3 genes, as well as insights into the evolution of this gene family in four Rosaceae species. Furthermore, high level of microsynteny in the four Rosaceae plants suggested that a largescale genome duplication event in EIL/EIN3 gene family was predated to speciation.

Introduction

Rosaceae species such as pear (Pyrus bretschneideri), mei (Prunus mume), peach (Prunus persica) and

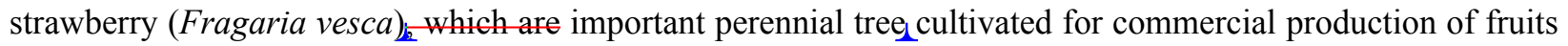


44 available worldwide. According to previous studies, the genomes of strawberry $(X=7)$, mei $(X=8)$, peach $(X$

$45=8)$, and pear $(X=17)$ shared an ancestor, which had 9 pairs of chromosomes (Shulaev et al. 2011 ; Verde et al.

46 2013; Wu et al. 2013; Zhang et al. 2012). Recently, the researchers confirmed that chromosome inversions,

47 fusions, and translocations played an important role in the evolution of the Rosaceae genome (Illa et al. 2011).

48 Some extant "diploid" species of Rosaceae family are originated from their polyploid ancestors, others are

49 actually thought to be true polyploids (Wendel 2000). These studies indicate that the diploid species in

50 Rosaceae have evolved with a complex history. There are several gene families which share highly conserved

51 genome sequences with each other among the related species of family Rosaceae, as well as other taxonomic

52 families. In this study, the EIL/EIN3 gene family was selected to investigate the specific evolutionary

relationships among the related species of family Rosaceae.

The EIN3/EIL gene family is a relatively small one in higher plants. Some EIN3/EIL genes have been

isolated from Arabidopsis thaliana (Chao et al. 1997a), tobacco (Kosugi \& Ohashi 2000; Rieu et al. 2003),

banana (Jourda et al. 2014), tomato (Tieman et al. 2001; Yokotani et al. 2003) and rice (Hiraga et al. 2009;

Mao et al. 2006). These plant-specific EIN3/EIL proteins are located in the nuclei, with the highly conserved

amino acid sequences at the N-termini, including several important structural features, such as acidic amino

acid regions, proline-rich regions and 5-basic amino acid clusters (BD I-V) (Chao et al. 1997a). Compared to the N-terminal sequences, the conservation of their C-termini is lower. For example, it was found that although 
Functions of the EIN3/EIL gene family have been studied in several plants, such as Hevea brasiliensis

(Yang et al. 2014) and tomato (Tieman et al. 2001; Yokotani et al. 2003). Recently, a number of researches on application of comparative genome in the analysis of evolution and function of gene family have been reported. For example, based on the comparative genomic analysis, Wang Yiyi et al (2015) explored the evolution and functional differences of WRKY type-III transcription factor family of poplar, grape, Arabidopsis and rice (Wang et al. 2015). Qing jin et al (2016) explored the evolution of WRKY I subfamily in Gramineae (Jing et al. 2016). However, there is still lack of specific evolutionary relationships of the EIN3/EIL gene family in Rosaceae. To address this question, the evolutionary relationships and gene duplication events of EIN3/EIL genes from Rosaceae species, including pear (Pyrus bretschneideri), mei (Prunus mume), peach (Prunus persica) and strawberry (Fragaria vesca), were analyzed, based on their phylogenetic relationships, microsynteny and environmental selection pressures analysis. In addition, the expression patterns of pear EIN3

/ EIL genes were investigated on a variety of organs/tissues including fruits at several developmental stages.

The results obtained from this study provided valuable information about EIN3/EIL genes that will aid future functional research involved in many important biological processes of this important gene family in flowering plants, especially in pear.

\section{Materials and methods}

\section{Sequence identification and collection}

The genome data of four Rosaceae species were obtained from their respective genome sequence websites: Pyrus bretschneideri from the GigaDB database (http://gigadb.org/site/index); Prunus mume from the Genome Database for Rosaceae (http://www.rosaceae.org/); Prunus persica from the Phytozome database 
86 (https://phytozome.jgi.doe.gov/pz/portal.html) and Fragaria vesca from the Joint Genome Institute

87 (http://www.jgi.doe.gov/). The Hidden Markov Model (HMM) profiles of EIN3/EIL domain (PF04873)

88 (Chang \& Shockey 1999; Chao et al. 1997a) were obtained from the Pfam database (http://pfam.xfam.org)

89 (Finn et al. 2006). The EIN3/EIL domain was used as query sequences to identify EIN3/EIL genes in four

90 Rosaceae species by using DNAtools software (E-value <0.001). To verify the EIN3/EIL genes in four

91 Rosaceae genomes, all putative proteins were validated by searching for the EIN3/EIL domain using InterPro

92 online tool (http://www.ebi.ac.uk/interpro/) (Zdobnov \& Apweiler 2001) and SMART database

93 (http://smart.embl-heidelberg.de/) (Letunic et al. 2012). In our study, only the EIN3/EIL domain-containing

94 sequences were retained.

95

96 Chromosomal location of EIN3/EIL genes

97

98

99

100

101

102

103

104

105

106

The genome annotation information was collected from GigaDB database (http://gigadb.org/site/index), Genome Database for Rosaceae (http://www.rosaceae.org/), Phytozome database (https://phytozome.jgi.doe.gov/pz/portal.html) and Joint Genome Institude (http://www.jgi.doe.gov/), respectively. Subsequently, the MapInspect software (http://mapinspect.software.informer.com/) was used for data visualization.

\section{Gene structure and motif analysis}

The exon-intron structure of each EIN3/EIL gene was determined by alignment of its CDS and genomic DNA sequence. Then a diagram was constructed using Structure Display Server website (Hu et al. 2014). Subsequently, the Online MEME server was used to screen the conserved motifs encoded by EIN3/EIL genes. 
107 108 109

110

111

112 113

Additionally, Pfam website (Punta et al. 2011) and SMART tools (Letunic et al. 2012) were used to annotate these structural motifs.

\section{Phylogenetic analysis of EIN3/EIL genes}

EIN3/EIL sequences were aligned using ClustalX version 1.83 (Thompson et al. 1997) and evolutionary relationships were inferred by analyzing an unrooted phylogenetic tree using MEGA 5 and neighbor-joining (NJ) method (Tamura et al. 2011) with the following parameters: poisson correction, pairwise deletion and 1000 bootstrap replicate.

\section{Microsynteny analysis}

In order to reveal the sequence features of the EIN3/EIL gene-containing regions, microsynteny analysis was performed across the four Rosaceae species (Pyrus bretschneideri, Prunus mume, Prunus persica and Fragaria vesca), using MCScanx (Multiple Collinearity Scan toolkit) (Wang et al. 2012) with the gene identifier file, the gene list file and the coding sequence file. Subsequently, a syntenic block was defined as a region containing three or more conserved homologs which were located within 100-kb downstream and upstream of protein-coding sequences.

\section{Environmental selection pressure analysis}

The nucleotide coding sequences from segmentally duplicated pairs were aligned by Clustal X (Thompson et al. 1997). Then DnaSP (version 5.10) was used to calculate the nonsynonymous (Ka) and synonymous (Ks) substitution rates of the homologues (Librado \& Rozas 2009). For each pair of duplicated regions, we 
128

129

130

131

132

133

134

135

136

137

138

139

140

141

142

143

144

145

146

147

148

estimated the mean Ks values of the flanking conserved genes for individual homologs. To further understand the selective pressure experienced by EIN3/EIL genes, $\mathrm{Ka}$, Ks and $\mathrm{Ka} / \mathrm{Ks}$ ratios were estimated using sliding window (with parameters: window size, $150 \mathrm{bp}$; step size, $9 \mathrm{bp}$ ) over the entire aligned length (Cao et al. 2016a; Han et al. 2016).

EIN3/EIL gene expression analysis in pear different tissues

To verify the expression patterns of EIN3/EIL genes, the qRT-PCR analysis was carried out. The first-strand cDNA was synthesized with Oligo18dT primer (Table S1) by using M-MLV reverse transcriptase (TakaRa, Japan) following the manufacture introduction. The TransStart Tip Green qPCR SuperMix (TransGen Biotech, China) with SYBR Green I as the fluorescent dye was used for the qPCR employing a Bio-rad CFX96 Touch ${ }^{\mathrm{TM}}$ Deep Well Real-Time PCR Detection system (BioRad, USA). The relative transcript level relative to the Pyrus tubulin gene (Wu et al. 2012) were estimated according to a previous workflow (Cao et al. 2016a; Cao et al. 2016b). For each sample, three replicates were set up in parallel experiments.

\section{Results and discussion}

\section{Identification of EIN3/EIL genes in Rosaceae}

The genome data of pear ( $P$. bretschneideri), mei (P. mume), peach ( $P$. persica) and strawberry $(F$. vesca) were recently published, respectively (Shulaev et al. 2011; Verde et al. 2013; Wu et al. 2013; Zhang et al. 2012). To identify the members of the EIN3/EIL gene families in these species, EIN3/EIL specific domain (PF04873) was used to perform Blastp searches of the local protein databases. Sequences identified were verified for EIN3/EIL domains through SMART database and InterPro online tool. Totally, 24 of EIN3/EIL genes were identified, including 10 in pear, 4 in peach, 5 in mei and 5 in strawberry, and named as PbEIL1- 
PbEIL10, PpEIL1-PpEIL4, PmEIL1-PmEIL5 and FvEIL1-FvEIL5, according to their locations in chromosome,

150

151

152

respectively (Table 1 and Figure 1). This result suggested that EIN3/EIL gene family was relatively small compared to other gene families in the studied species. Similar indication was also reported by the previous studies in which 6, 5, 4, 6 and 17 EIN3/EILs genes were found in Arabidopsis thaliana (Chao et al. 1997a; Guo \& Ecker 2004), tobacco (Kosugi \& Ohashi 2000; Rieu et al. 2003), tomato (Tieman et al. 2001; Yokotani et al. 2003), rice (Hiraga et al. 2009; Mao et al. 2006) and banana (Jourda et al. 2014), respectively. Furthermore, it was found that the genome sizes and number of EIN3/EIL gene family members appeared not to have a direct relevance. For example, although there was no significant variety in genome size of pear $(271.9 \mathrm{Mb})$ and strawberry $(240 \mathrm{Mb})$, the number of EIN3/EIL genes obviously changed. Contrarily, the number of EIN3/EIL genes of the peach $(224.6 \mathrm{Mb})$ and strawberry $(240 \mathrm{Mb})$ had a corresponding relationship with their genome size. Remarkably, compared with those in peach, mei and strawberry, the numbers of EIN3/EIL genes in pear were found to be almost doubled. Moreover, the chromosome numbers of peach, mei and strawberry are 16, 16 and 14, respectively (Shulaev et al. 2011; Verde et al. 2013; Wu et al. 2013; Zhang et al. 2012), whereas the chromosome number of pear is 34 , indicating that the EIN3/EIL gene family has undergone an expansion corresponding to the variation in chromosome number. However, a recent whole genome duplication event (30-45 million years ago) (Wu et al. 2013) occurred in pear but not in peach, mei and strawberry, probably contributed to the expansion of EIN3/EIL gene family in the pear.

To determine the distribution of EIN3/EIL genes on chromosomes among pear, peach, mei and strawberry, respectively, a chromosome map (Figure 1) was drawn based on genome annotation (Wu et al. 2013). In pear, two EIN3/EIL genes were located on chromosome 2, and one gene on chromosome 3, 8, 11 and 15, respectively, with the remaining genes localized on different scaffold regions (Figure 1C). In peach, two 
170

171

172

173

174

175

176

177

178

179

180

181

182

183

184

185

186

187

188

189

190

EIN3/EIL genes were found on chromosome 2 and 6, respectively (Figure 1D). In mei, two EIN3/EIL genes

were distributed on chromosome 1 and 5, respectively, with the remaining one localized on a scaffold region

(Figure 1B). And in strawberry, two EIN3/EIL genes were distributed on chromosome 1 and 7, respectively, with the remaining one localized on chromosome 3 (Table 1 and Figure 1A).

\section{Phylogenetic analysis of EIN3/EIL genes}

The phylogenetic tree containing EIN3/EIL gene homologs from a variety of species, including Arabidopsis thaliana, rice, banana, sorghum, maize, Brachypodium distachyon, Thellungiella parvula, and four Rosaceae species, was also constructed (Figure S1). As shown in the phylogenetic tree, most EIN3/EIL genes from four Rosaceae species were clustered together. To further understand the evolutionary history of EIN3/EIL genes in Rosaceae, phylogenetic analysis was carried out using neighbor joining (NJ) method. As shown in Figure 2, 24

EIN3/EIL sequences were divided into two subfamilies, designated as A and B, which contained four classes (Classes A1, A2, B1 and B2). Classes A1, B1 and B2 were composed of the EIN3/EIL genes from the four species (pear, peach, mei and strawberry), while the Class A2 contained the members only from pear. According to our study, a whole genome duplication event happened 30-45 million years ago in pear, but not in peach, yang mei and strawberry (Wu et al. 2013). This result suggested the probable reason for occurrence of Class A2 genes in pear. Remarkably, EIN3/EIL genes from peach and mei showed higher similarity with each other according to genetic distance, which was consistent with a previous study reporting that the closer relationship between peach and mei versus peach and pear/ strawberry (Cao et al. 2016a; Cao et al. 2016b; Cao et al. 2016d).

Each of the four Rosaceae species contributed at least one members of EIN3/EIL gene to each class, with 
191

192

193

194

195

196

197

198

199

200

201

202

203

204

205

206

207

208

209

210

211

the exception for Class A2 (Figure 2). Therefore, we deduced that EIN3/EIL genes had rapidly been duplicated

before these dicotyledon species diverged. However, only the EIN3/EIL genes in class A2 revealed an internal duplication. In addition, we identified three pairs of orthologous genes among the EIN3/EIL genes: PmEIL3 and PpEIL1, PpEIL2 and PmEIL4, PpEIL3 and PmEIL5 based on the phylogenetic analysis.

\section{Structural analysis of EIN3/EIL genes}

Previous studies have suggested that gene structural diversity is the primary resource for the evolution of multigene families (Cao et al. 2016c; Leitch \& Leitch 2013; Mercereau-Puijalon et al. 2002). To characterize the structural diversity of the EIN3/EIL gene family, exon-intron organization of each EIN3/EIL genes was analyzed. As shown in Figure 3A, most genes did not contain introns, such as FvEIL1, PbEIL1, PmEIL3 and PpEIL4 et al. Furthermore, PbEIL 7 contained 8 introns, followed by FvEIL3 (5), whereas FvEIL5 had three introns, PmEIL2 had two introns and 8 EIN3/EIL genes contained one intron (Figure 3A). These results implied that the intron/exon loss and acquire has occurred in the evolution of the EIN3/EIL gene family, which may be able to explain the functional divergence of closely related EIN3/EIL genes. In present study, the gene structures of the EIN3/EIL homologous gene pairs were investigated. We found the exon number of two gene pairs (PbEIL2/PbEIL8 and PmEIL1/PmEIL2) had changed. Further analysis indicated that PbEIL8 and PmEIL2 obtained one exon during evolution, while PbEIL2 and PmEIL1 lost one exon. These diversities might be due to single intron loss or obtain events during evolution.

Because 24 EIN3/EIL genes did not have high similarity, MEME web server was used to find conserved motifs. Subsequently, we identified twenty conserved motifs, which were shown in Table S2 and Figure 3B.

The Pfam and SMART database were used to annotate the individual of the putative motifs. Motif 1 and motif 
2122 were identified to encode a conserved EIN3/EIL domain (Chang \& Shockey 1999; Chao et al. 1997a),

213 whereas the remaining motifs did not get function annotation. Most EIN3/EIL proteins have motifs 1, 2, 4, 7,

21410 and 19. In addition, several proteins from clade B2 contained unique motif 12, which might imply its

215 specific functions. Remarkably, most of the closely related EIN3/EIL proteins in the same clade exhibited

216 similar motif compositions (e.g. PmEIL5/PpEIL3 and PbEIL3/PbEIL5), indicating their functional similarity

217 among these EIN3/EIL proteins. Summarily, the similarity in motif distribution and exon-intron structure of

218 most EIN3/EIL proteins supported the results from phylogenetic analysis of the EIN3/EIL genes, whereas the

219 differences of the related characteristics in the different classes indicated that their functions were diversified.

220

221

Conserved microsynteny of EIN3/EIL genes in the four Rosaceae species

222

223

224

225

226

227

228

229

230

231

232

Based on the whole-genome data, species microsynteny can be used to identify the location of orthologous genes and/or paralogous genes (Cao et al. 2016d; Lin et al. 2014). To identify the homologous genes (orthology or paralogy) within the EIN3/EIL genes from four Rosaceae species (pear, peach, mei and strawberry), as well as their evolutionary history, microsynteny analysis was performed. By pairwise comparisons of flanking sequences in the chromosomal regions containing EIN3/EIL genes, three or more pairs were present in this region, which were considered as either conserved microsynteny or high levels of microsynteny.

In this study, a total of 55 flanking sequences containing EIN3/EIL genes could be assembled into 28 regions and divided into seven microsynteny groups. It was supposed that EIN3/EIL genes from the same group should evolve from the most recent common ancestor. Based on this criterion, orthology and/or paralogy relationships, as well as their evolutionary origins, were detected among EIN3/EIL genes of the four Rosaceae 
233 species. Nine, five, four and four out of the seven microsynteny groups are from pear, peach, mei and

234 strawberry, respectively (Figure 4). In class B2, two gene pairs (PbEIL1 and PbEIL9, PbEIL9 and PbEIL10)

235 with both a higher level of microsynteny and a noticeable inverted duplication, were identified. Interestingly, it

236 was also found that some duplication rules in several regions were in disorder, such as $F v E I L 1$ and $F v E I L 2$,

237 FvEIL1 and PpEIL4 (Figure 4). Similar microsynteny was identified in other classes with a concordant

inverted microsynteny (Figure 4). In addition, according to the constructed phylogenetic tree, conservation of

microsynteny between different families appeared gradually. However, some flanking genes in each

microsyntenic group were not conserved, indicating they arose later than this duplication event (Figure 4).

Furthermore, we only identified four pairs of intraspecies microsynteny groups from pear (PbEIL1 and PbEIL9,

PbEIL3 and PbEIL5, PbEIL4 and PbEIL6, PbEIL9 and PbEIL10), but peach, mei and strawberry were

excluded (Figure 5). This difference might be resulted from the expansion of pear EIN3/EIL genes. However,

no similar gene expansion was identified in peach, mei and strawberry. Some previous studies has

hypothesized that transcription factors should be generally and preferentially retained after genome

duplications (Blanc \& Wolfe 2004), with a lower frequency of tandem duplication events in a number of

also strong evidences for it. 
254 highest value of synteny quality found between pear and peach was $33.29 \%$. And the lower value of synteny

255 quality was obtained between strawberry and peach (10.26\%) and mei (13.26\%) The relative synteny quality in

256 the pear/mei and pear/strawberry syntenic regions was $31.35 \%$ and $26.17 \%$, which was substantially lower

257 than $32.13 \%$ found in the pear/peach synteny blocks. Our results were essentially consistent with their evolutionary relationship (Xiang et al. 2016; Zhong et al. 2015).

\section{Strong purifying selection for $E I N 3 / E I L$ genes in four Rosaceae species}

261

262

263

264

265

266

267

268

269

270

271

272

273

274

In general, Ks values can be used to estimate evolutionary data of the whole genome duplication events or segmental duplication events. Previous studies showed that pear had experienced two whole genome duplication events, including an ancient whole genome duplication (Ks $\sim 1.5-1.8)$ estimated at 140 MYA (Fawcett et al. 2009) and a recent whole genome duplication (Ks $\sim 0.15-0.3$ ) estimated at 30-45 MYA (Wu et al. 2013)), while peach, mei and strawberry only experienced an ancient whole genome duplication event.

Therefore, Ks values were applied to analyze the whole genome duplication or segmental duplication events in EIN3/EILs of four Rosaceae species. As shown in Table S3, the mean Ks values of each duplication pairs in the syntenic region were lists. In pear, we found the mean Ks values of EIN3/EIL gene pairs were 0.0363, 0.1717 and 0.2836 , respectively. It was obvious that these duplications might be resulting from the latest whole genome duplication (30-45 MYA; Ks $\sim 0.15-0.3$ ), but an ancient whole genome duplication ( 140MYA; Ks $\sim 1.5-1.8)$ in pear.

In addition, the $\mathrm{Ka} / \mathrm{Ks}$ values are widely used to represent the gene selection pressure and evolution rate [40]: $\mathrm{Ka} / \mathrm{Ks}$ value with $>1$ indicates positive selection with accelerated evolution, $\mathrm{Ka} / \mathrm{Ks}<1$ indicates negative/purifying selection with the functional constraint, and $\mathrm{Ka} / \mathrm{Ks}=1$ suggests that the genes are drifting 
275 neutrally. In this study, all paralogs was found with $\mathrm{Ka} / \mathrm{Ks}$ ratios $<1$ (Figure 6), indicating their purifying

276 selection. Furthermore, to better understand the delineate regions of diversifying and purifying selection in the

$277 E I N 3 / E I L$ gene family, a sliding window analysis of the $\mathrm{Ka} / \mathrm{Ks}$ values between paralogs was performed (Figure

278 4), the EIN3/EIL domains in the N-termini exhibited stronger purifying selection compared with the whole

279 gene regions (C-termini). These results suggested that the EIN3/EIL genes had undergone strongly purifying

280 selection, especially for EIN3/EIL domains in the N-termini (Figure 4Q). Overall, strong evolutionary

281 constraints were involved in EIN3/EIL gene evolution, which may contribute to their functional stability. On

282 the other hand, some parts of protein-coding genes had undergone positive selection, implying the generation

283 of innovative gene functions.

\section{Expression profiles analysis of $P b E I L$ genes in different tissues}

To increase our understanding the potential functions of pear EIN3/EIL genes during development, the qRT-

PCR analysis was carried out to determine the expression profiles of $10 \mathrm{PbEIL}$ genes in different tissues. As

shown in Figure 7 and Table S4, 10 pear EIN3/EIL genes showed significantly different tissue-specific

expression patterns in eight samples from root, stem, leaves and fruits in several development stages. Among

the 10 pear EIN3/EIL genes, three (PbEIL5, PbEIL6 and PbEIL10) showed the highest transcript accumulation

in the leaves, three (PbEIL2, PbEIL3 and PbEIL9) in 145 DAF (days after flowering), two (PbEIL1 and

PbEIL4) in 79 DAF, one (PbEIL7) in roots. Additionally, the duplication gene pairs showed different 
296 297 298 299 300

301

302

303

304

305

306

307

308

309

310

311

312

313

314

315

316

317

318

subfunctionalization after duplication. At the same time, this phenomenon was also observed among other EIN3/EIL duplication genes (Jourda et al. 2014).

\section{Conclusions}

In this study, we identified 24 EIN3/EIL genes from four Rosaceae species (pear, peach, mei and strawberry).

Subsequently, a systematic analysis, including their chromosomal location, evolutionary relationship, conserved microsynteny, gene structure and sliding window, was carried out. According to phylogenetic analysis, the EIN3/EIL genes divided into four classes. Remarkably, high level of microsynteny of EIL/EIN3 family in Rosaceae was found, indicating that the genome duplication plays a key role in the expansion of the EIL/EIN3 genes in the Rosaceae. In these EIL/EIN3 genes, all paralogs have experienced purifying selection, especially the EIL/EIN3 domains in the Rosaceae. Furthermore, the expression profiles of the PbEIL genes suggested that the recent whole genome duplication derived genes show indications of subfunctionalization. These results may help promote the extrapolation of EIL/EIN3 gene functions in future.

\section{ACKNOWLEDGEMENTS}

We would like to thank Muhammad Abdullah for his careful reading and helpful comments on this manuscript. We extend our thanks to the reviewers and editors for their careful reading and helpful comments on this manuscript.

\section{References}

Blanc G, and Wolfe KH. 2004. Widespread paleopolyploidy in model plant species inferred from age distributions of duplicate genes. Plant Cell 16:1667-1678.

Cannon SB, Mccombie WR, Sato S, Tabata S, Denny R, Palmer L, Katari M, Young ND, and Stacey G. 2003. Evolution and microsynteny of the apyrase gene family in three legume genomes. Molecular Genetics and Genomics 
270:347-361.

Cannon SB, Sterck L, Rombauts S, Sato S, Cheung F, Gouzy J, Wang X, Mudge J, Vasdewani J, and Schiex T. 2006. Legume genome evolution viewed through the Medicago truncatula and Lotus japonicus genomes. Proceedings of the National Academy of Sciences of the United States of America 103:14959-14964.

Cao Y, Han Y, Li D, Lin Y, and Cai Y. 2016a. MYB Transcription Factors in Chinese Pear (Pyrus bretschneideri Rehd.): Genome-Wide Identification, Classification, and Expression Profiling during Fruit Development. Frontiers in Plant Science 7.

Cao Y, Han Y, Li D, Lin Y, and Cai Y. 2016b. Systematic Analysis of the 4-Coumarate:Coenzyme A Ligase (4CL) Related Genes and Expression Profiling during Fruit Development in the Chinese Pear. Genes 7:89.

Cao Y, Han Y, Meng D, Li D, Jin Q, Lin Y, and Cai Y. 2016c. Structural, Evolutionary, and Functional Analysis of the Class III Peroxidase Gene Family in Chinese Pear (Pyrus bretschneideri). 7.

Cao YP, Han Y, Jin Q, Lin Y, and Cai Y. 2016d. Comparative genomic analysis of the GRF genes in Chinese pear (Pyrus bretschneideri Rehd), poplar (Populous), grape (Vitis vinifera), Arabidopsis and rice (Oryza sativa). Frontiers in Plant Science 7. 10.3389/fpls.2016.01750

Chang C, and Shockey JA. 1999. The ethylene-response pathway: signal perception to gene regulation. Current opinion in plant biology 2:352-358.

Chao Q, Rothenberg M, Solano R, Roman G, Terzaghi W, and Ecker JR. 1997a. Activation of the ethylene gas response pathway in Arabidopsis by the nuclear protein ETHYLENE-INSENSITIVE3 and related proteins. Cell 89:1133-1144.

Chao Q, Rothenberg M, Solano R, Roman G, Terzaghi W, and Ecker JR. 1997b. Activation of the ethylene gas response pathway in Arabidopsis by the nuclear protein ETHYLENE-INSENSITIVE3 and related proteins. Cell 89:1133-1144.

Fawcett JA, Maere S, and Van dPY. 2009. Plants with double genomes might have had a better chance to survive the Cretaceous-Tertiary extinction event. Proceedings of the National Academy of Sciences 106:5737-5742.

Finn RD, Mistry J, Schuster-Böckler B, Griffiths-Jones S, Hollich V, Lassmann T, Moxon S, Marshall M, Khanna A, and Durbin R. 2006. Pfam: clans, web tools and services. Nucleic acids research 34:D247-D251.

Freeling M. 2009. Bias in plant gene content following different sorts of duplication: tandem, whole-genome, segmental, or by transposition. Annual Review of Plant Biology 60:433-453.

Guo H, and Ecker JR. 2004. The ethylene signaling pathway: new insights. Curr Opin Plant Biol 7:40-49. Current opinion in plant biology 7:40-49.

Han Y, Ding T, Su B, and Jiang H. 2016. Genome-Wide Identification, Characterization and Expression Analysis of the Chalcone Synthase Family in Maize. International journal of molecular sciences 17:161.

Hiraga S, Sasaki K, Hibi T, Yoshida H, Uchida E, Kosugi S, Kato T, Mie T, Ito H, and Katou S. 2009. Involvement of two rice ETHYLENE INSENSITIVE3-LIKE genes in wound signaling. Molecular Genetics and Genomics 282:517529.

Hu B, Jin J, Guo YA, Zhang H, Luo J, and Gao G. 2014. GSDS 2.0: an upgraded gene feature visualization server. Bioinformatics 31:1296.

Illa E, Sargent DJ, Girona EL, Bushakra J, Cestaro A, Crowhurst R, Pindo M, Cabrera A, van der Knaap E, and lezzoni A. 2011. Comparative analysis of rosaceous genomes and the reconstruction of a putative ancestral genome for the family. BMC Evolutionary biology 11:1. 
Jing J, Kong J, Qiu J, Zhu H, Peng Y, and Jiang H. 2016. High level of microsynteny and purifying selection affect the evolution of WRKY family in Gramineae. Development Genes \& Evolution 226:15-25.

Jourda C, Cardi C, Mbéguiéambéguié D, Bocs S, Garsmeur O, D'Hont A, and Yahiaoui N. 2014. Expansion of banana (Musa acuminata) gene families involved in ethylene biosynthesis and signalling after lineage-specific whole-genome duplications. New Phytologist 202:986-1000.

Kosugi S, and Ohashi Y. 2000. Cloning and DNA-binding properties of a tobacco Ethylene-Insensitive3 (EIN3) homolog. Nucleic acids research 28:960-967.

Lee JH, and Kim WT. 2003. Molecular and biochemical characterization of VR-EILs encoding mung bean ETHYLENE INSENSITIVE3-LIKE proteins. Plant Physiology 132:1475-1488.

Leitch IJ, and Leitch AR. 2013. Genome Size Diversity and Evolution in Land Plants.

Letunic I, Doerks T, and Bork P. 2012. SMART 7: recent updates to the protein domain annotation resource. Nucleic acids research 40:D302-D305.

Librado P, and Rozas J. 2009. DnaSP v5: a software for comprehensive analysis of DNA polymorphism data. Bioinformatics 25:1451-1452.

Lin Y, Cheng Y, Jin J, Jin X, Jiang H, Yan H, and Cheng B. 2014. Genome Duplication and Gene Loss Affect the Evolution of Heat Shock Transcription Factor Genes in Legumes. PLoS One 9:e102825.

Lynch M, and Conery JS. 2000. The evolutionary fate and consequences of duplicate genes. Science 290:1151-1155.

Mao C, Wang S, Jia Q, and Wu P. 2006. OsEIL1, a rice homolog of the Arabidopsis EIN3 regulates the ethylene response as a positive component. Plant molecular biology 61:141-152.

Mercereau-Puijalon O, Barale JC, and Bischoff E. 2002. Three multigene families in Plasmodium parasites: facts and questions. International Journal for Parasitology 32:1323-1344.

Punta M, Coggill PC, Eberhardt RY, Mistry J, Tate J, Boursnell C, Pang N, Forslund K, Ceric G, and Clements J. 2011. The Pfam protein families database. Nucleic acids research:gkr1065.

Rieu I, Mariani C, and Weterings K. 2003. Expression analysis of five tobacco EIN3 family members in relation to tissue - specific ethylene responses. Journal of Experimental Botany 54:2239-2244.

Shulaev V, Sargent DJ, Crowhurst RN, Mockler TC, Folkerts O, Delcher AL, Jaiswal P, Mockaitis K, Liston A, and Mane SP. 2011. The genome of woodland strawberry (Fragaria vesca). Nature genetics 43:: 109-116.

Tamura K, Peterson D, Peterson N, Stecher G, Nei M, and Kumar S. 2011. MEGA5: molecular evolutionary genetics analysis using maximum likelihood, evolutionary distance, and maximum parsimony methods. Molecular biology and evolution 28:2731-2739.

Thompson JD, Gibson TJ, Plewniak F, Jeanmougin F, and Higgins DG. 1997. The CLUSTAL_X windows interface: flexible strategies for multiple sequence alignment aided by quality analysis tools. Nucleic acids research 25:4876-4882.

Tieman DM, Ciardi JA, Taylor MG, and Klee HJ. 2001. Members of the tomato LeEIL (EIN3 - like) gene family are functionally redundant and regulate ethylene responses throughout plant development. The Plant Journal 26:47-58.

Verde I, Abbott AG, Scalabrin S, Jung S, Shu S, Marroni F, Zhebentyayeva T, Dettori MT, Grimwood J, and Cattonaro F. 2013. The high-quality draft genome of peach (Prunus persica) identifies unique patterns of genetic diversity, domestication and genome evolution. Nature genetics 45:487-494.

Waki K, Shibuya K, Yoshioka T, Hashiba T, and Satoh S. 2001. Cloning of a cDNA encoding EIN3 - like protein (DC - EIL1) and decrease in its mRNA level during senescence in carnation flower tissues. Journal of 
401

402

403

404

405

406

407

408

409

410

411

412

413

414

415

416

417

418

419

420

421

422

423

424

425

426

427

428

Experimental Botany 52:377-379.

Wang Y, Feng L, Zhu Y, Li Y, Yan H, and Xiang Y. 2015. Comparative genomic analysis of the WRKY III gene family in populus, grape, arabidopsis and rice. Biology direct 10:1.

Wang Y, Tang H, DeBarry JD, Tan X, Li J, Wang X, Lee T-h, Jin H, Marler B, and Guo H. 2012. MCScanX: a toolkit for detection and evolutionary analysis of gene synteny and collinearity. Nucleic acids research 40:e49-e49.

Wendel JF. 2000. Genome evolution in polyploids. Plant molecular evolution: Springer, 225-249.

Wu J, Wang Z, Shi Z, Zhang S, Ming R, Zhu S, Khan MA, Tao S, Korban SS, and Wang H. 2013. The genome of the pear (Pyrus bretschneideri Rehd.). Genome research 23:396-408.

Wu T, Zhang R, Gu C, Wu J, Wan H, Zhang S, and Zhang S. 2012. Evaluation of candidate reference genes for real time quantitative PCR normalization in pear fruit. Afr J Agric Res 7:3701-3704.

Xiang Y, Huang CH, Yi H, Wen J, Li S, Yi T, Chen H, Xiang J, and Hong M. 2016. Evolution of Rosaceae fruit types based on nuclear phylogeny in the context of geological times and genome duplication. Molecular Biology \& Evolution.

Yang ZP, Li HL, Guo D, and Peng SQ. 2014. Identification and characterization of the EIN3/EIL gene family in Hevea brasiliensis. Plant Physiology \& Biochemistry 11:1-11.

Yokotani N, Tamura S, Nakano R, Inaba A, and Kubo Y. 2003. Characterization of a novel tomato EIN3 - like gene (LeEIL4). Journal of Experimental Botany 54:2775-2776.

Zdobnov EM, and Apweiler R. 2001. InterProScan--an integration platform for the signature-recognition methods in InterPro. Bioinformatics 17:847-848.

Zhang Q, Chen W, Sun L, Zhao F, Huang B, Yang W, Tao Y, Wang J, Yuan Z, and Fan G. 2012. The genome of Prunus mume. Nature Communications 3:1318.

Zhong Y, Yin H, Sargent DJ, Malnoy M, and Cheng ZM. 2015. Species-specific duplications driving the recent expansion of NBS-LRR genes in five Rosaceae species. BMC genomics 16:1-16.

4 


\section{Figure 1 (on next page)}

Figure 1. Chromosomal location of EIN3/EIL genes in the genomes of strawberry (A), mei (B), pear (C) and peach (D).

The distribution of EIN3/EIL genes among the chromosomes in each species was diverse. The chromosome number was represented at the top of each chromosome. The left scale indicates the megabases $(\mathrm{Mb})$. 


\section{Manuscript to be reviewed}
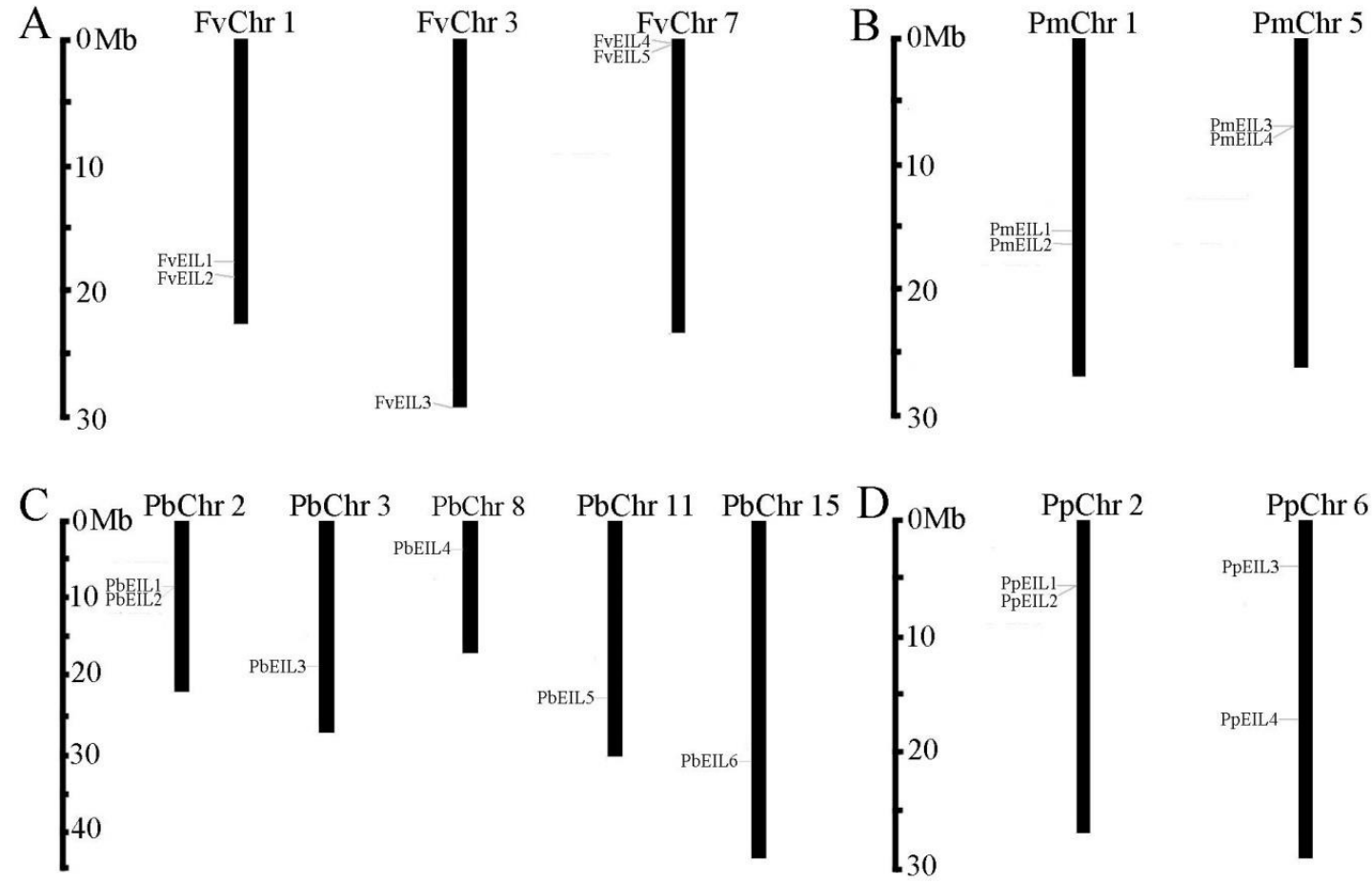


\section{Figure 2 (on next page)}

Figure 2. Phylogenetic tree of EIN3/EIL proteins from pear, peach, yangmei and strawberry. The neighbor-joining (NJ) tree was constructed by using MEGA 5 software.

The neighbor-joining (NJ) tree was constructed by using MEGA 5 software (1000 bootstrap replicates). The different colors suggest the different species background for each EIN3/EIL protein. Gene names are listed in Table 1. The scale bar represents 0.1 amino acid changes per site. 


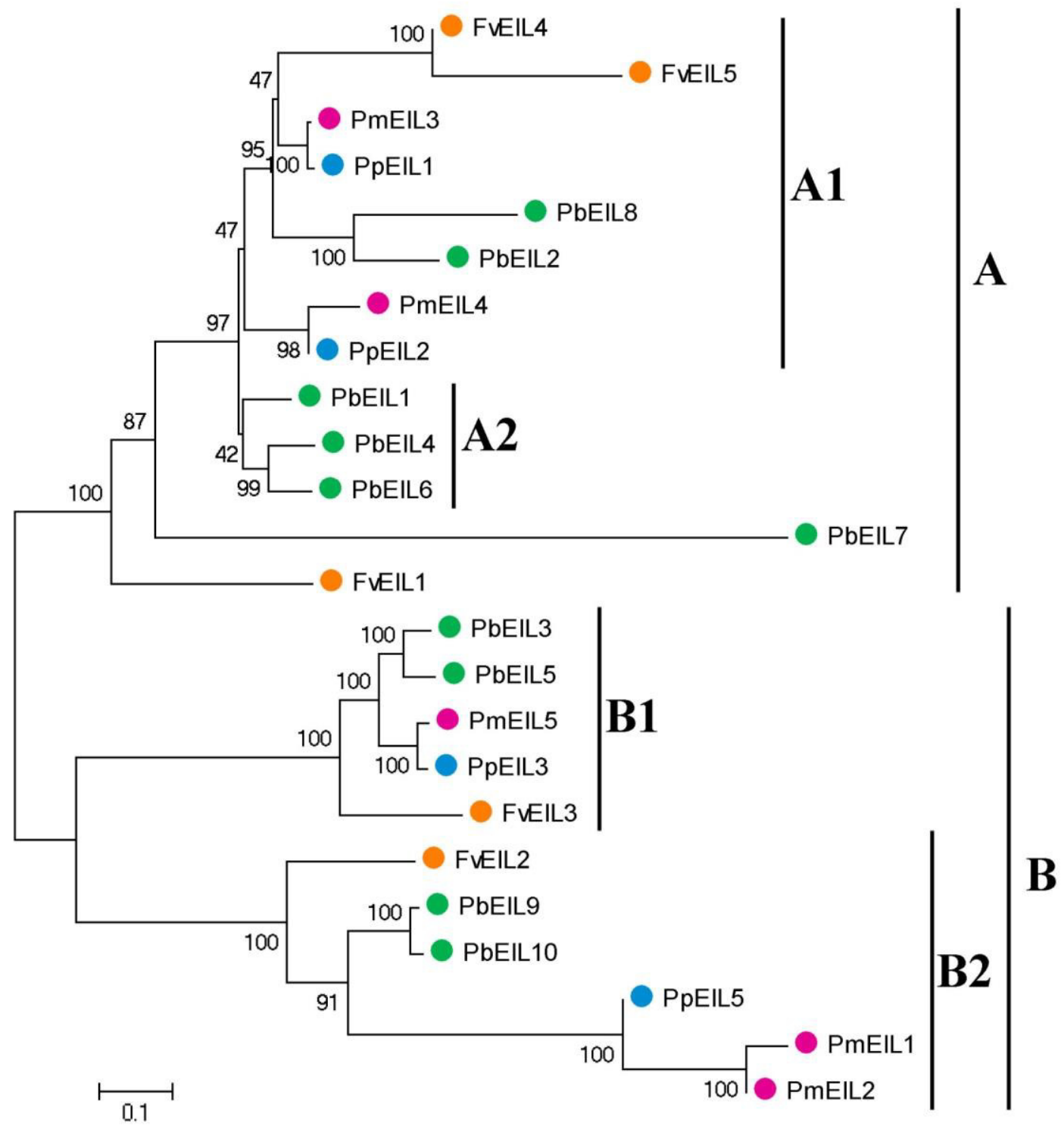




\section{Figure 3 (on next page)}

Figure 3. Gene structure (A) and conserved motif compositions (B) of EIN3/EIL genes in Rosaceae species.

Untranslated regions (UTRs), introns and exons are represented by blue boxes, thin lines and green rectangles, respectively. Note that the gene or protein lengths can be estimated by using the scale at the bottom. 
A

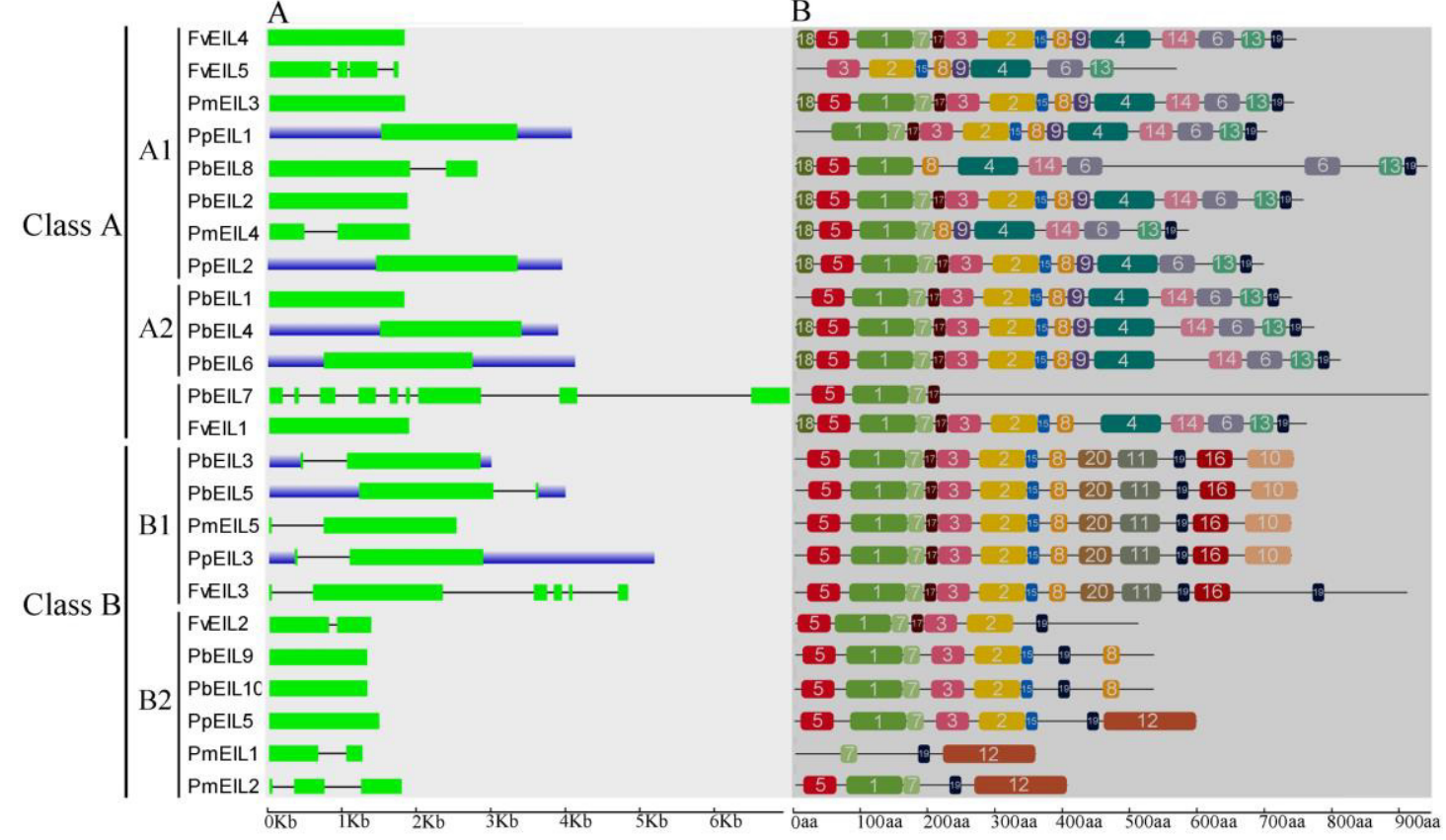




\section{Figure 4 (on next page)}

Figure 4. Interspecies microsynteny related to EIN3/EIL families in four Rosaceae.

The relative positions of all flanking protein-coding genes were defined by anchored EIN3/EIL genes, highlighted in red. The gene's orientations are shown as triangle, with gray lines corresponding to chromosomal segments. 
Pm chr5: 6.81-7.01 Mb Anchor gene: PmEIL3

Fv chr7: 12.61-12.81 Mb Anchor gene: FvEIL4

Pp chr2: 5.42-5.62 Mb Anchor gene: PpEILI $\mathrm{Pb}$ chr2: 8.67-8.87 Mb Anchor gene: PbEILI

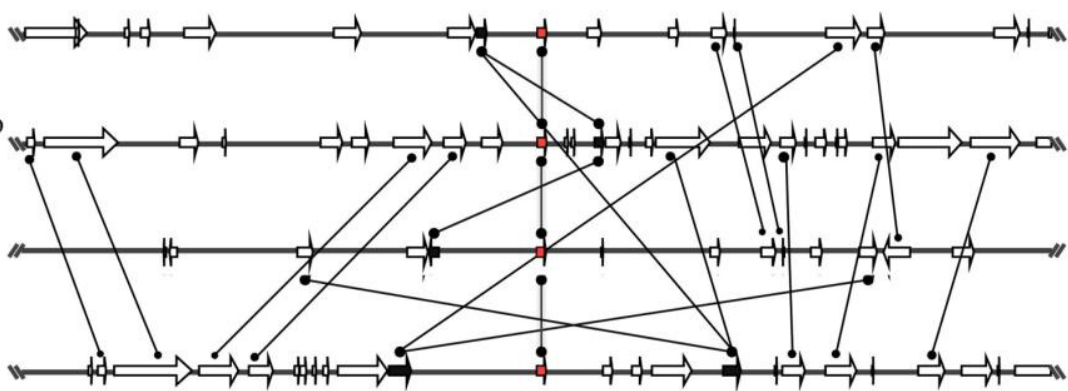

$\mathrm{Pb}$ chr3: $18.44-18.64 \mathrm{Mb}$ Anchor gene: PbEIL3

Fv chr3: $30.15-30.35 \mathrm{Mb}$ Anchor gene: FvEIL3 Pm sca103: 1.14-1.34 Mb Anchor gene: PmEIL5 Pb chr11: 14.04-18.24 Mb Anchor gene: PbEIL5 Pp chr6: $3.78-3.98 \mathrm{Mb}$ Anchor gene: PpEIL3

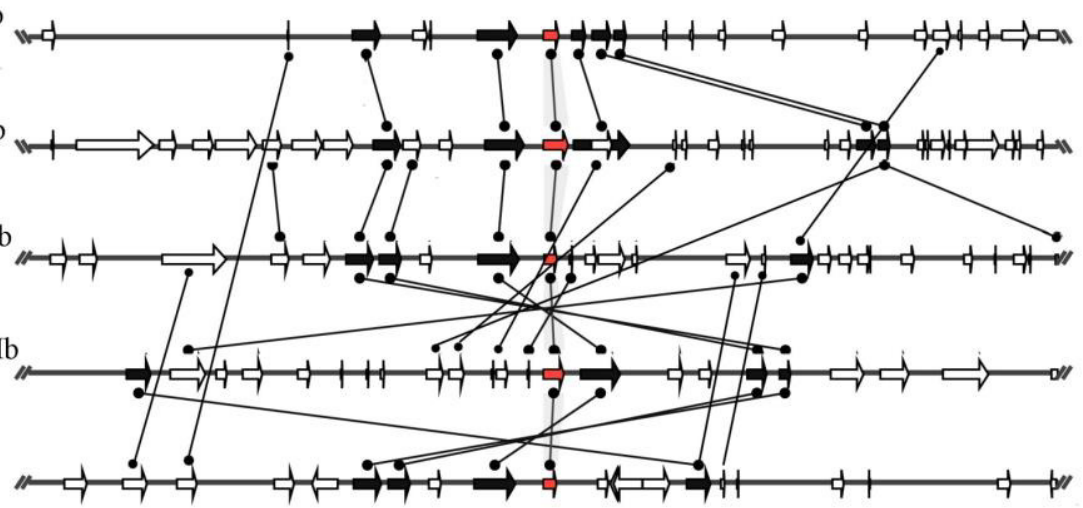

Pm chr1: 15.15-16.35 Mb

Anchor gene: PmEIL2

Pp chr8: 16.88-17.08 Mb Anchor gene: PpEIL4 Fv chr1: 18.79-18.99 Mb Anchor gene: FvEIL2 Pm ch1: 16.15-16.35 Mb Anchor gene: PmEIL2 $\mathrm{Pb}$ chr2: 8.67-8.87 Mb Anchor gene: PbEIL1 Pb sca341: 0.84-1.04 Mb Anchor gene: PbEIL9
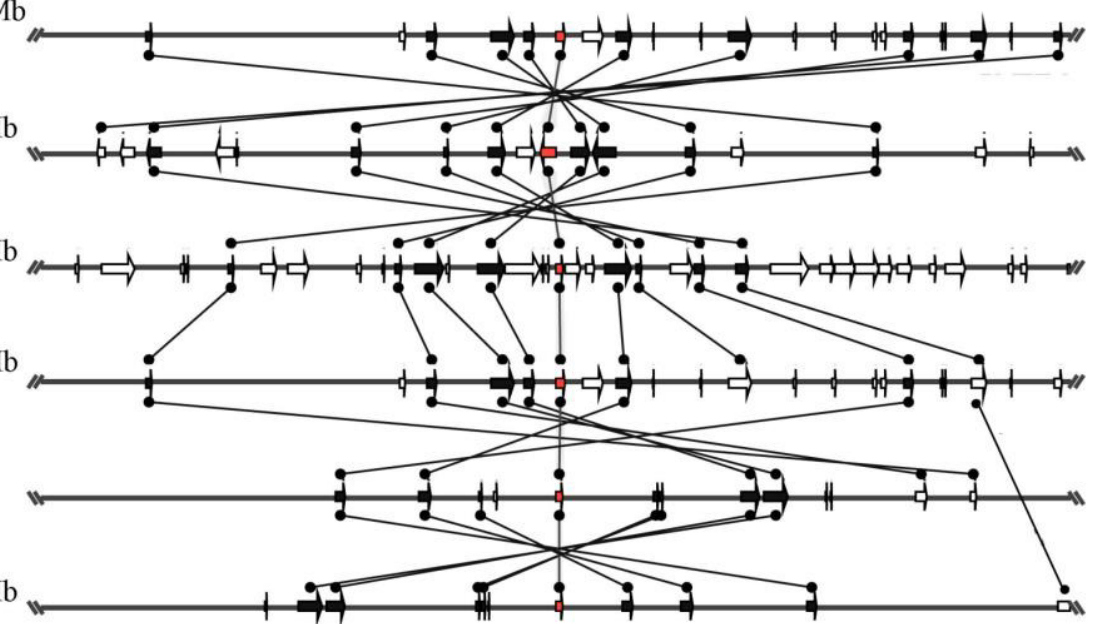

Fv chr1: 18.79-18.99 Mb "Anchor gene: FvEIL2 Pm chr1: 15.14-15.34 Mb Anchor gene: PmEILI Pp chr6: 16.88-17.08 Mb Anchor gene: PpEIL4 


\section{Figure $\mathbf{5}$ (on next page)}

Figure 5. Intraspecific microsynteny related to EIN3/EIL families with the same species.

The relative positions of all flanking protein-coding genes were defined by anchored EIN3/EIL genes, highlighted in red. The gene's orientations are shown as triangle, with gray lines corresponding to chromosomal segments. 
$\mathrm{Pb}$ chr15: 31.79-31.99 Mb Anchor gene: PbEIL6

Pb chr8: 3.59-3.79 Mb Anchor gene: PbEIL4

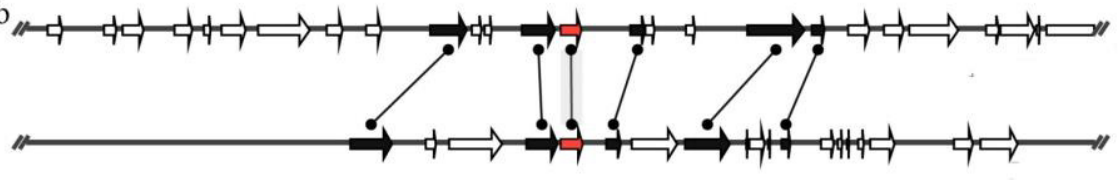

Pb chr11: 22.79-22.99 Mb Anchor gene: PbEIL5

$\mathrm{Pb}$ chr3: 18.71-18.91 Mb Anchor gene: PbEIL3

Pb scal70: 0.23-0.43 Mb Anchor gene: PbEIL9

Pb sca170: 0.35-0.45 Mb Anchor gene: PbEIL10
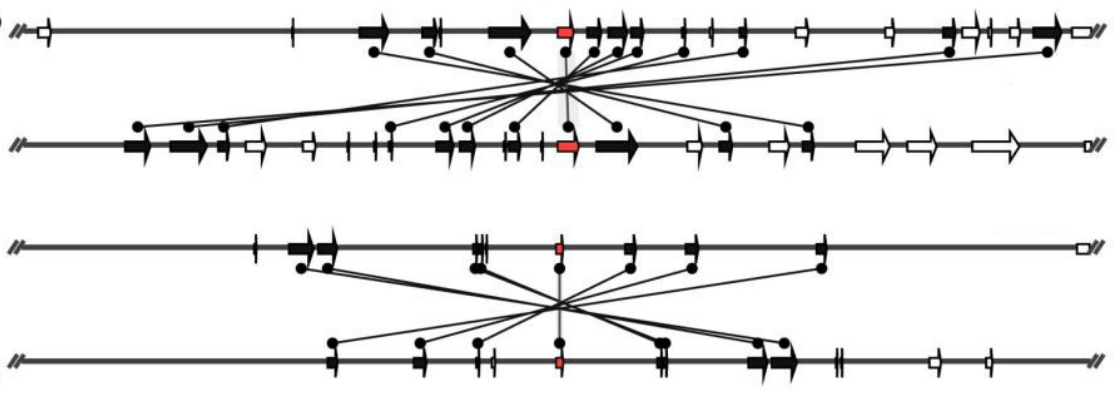


\section{Figure 6 (on next page)}

Figure 6. Sliding window plots of duplicated EIN3/EIL genes in Rosaceae species.

The gray blocks indicate the positions of the EIN3/EIL domains. The window size is $150 \mathrm{bp}$, and the step size is $9 \mathrm{bp}$. The $\mathrm{x}$-axis denotes the synonymous distances within each gene. 

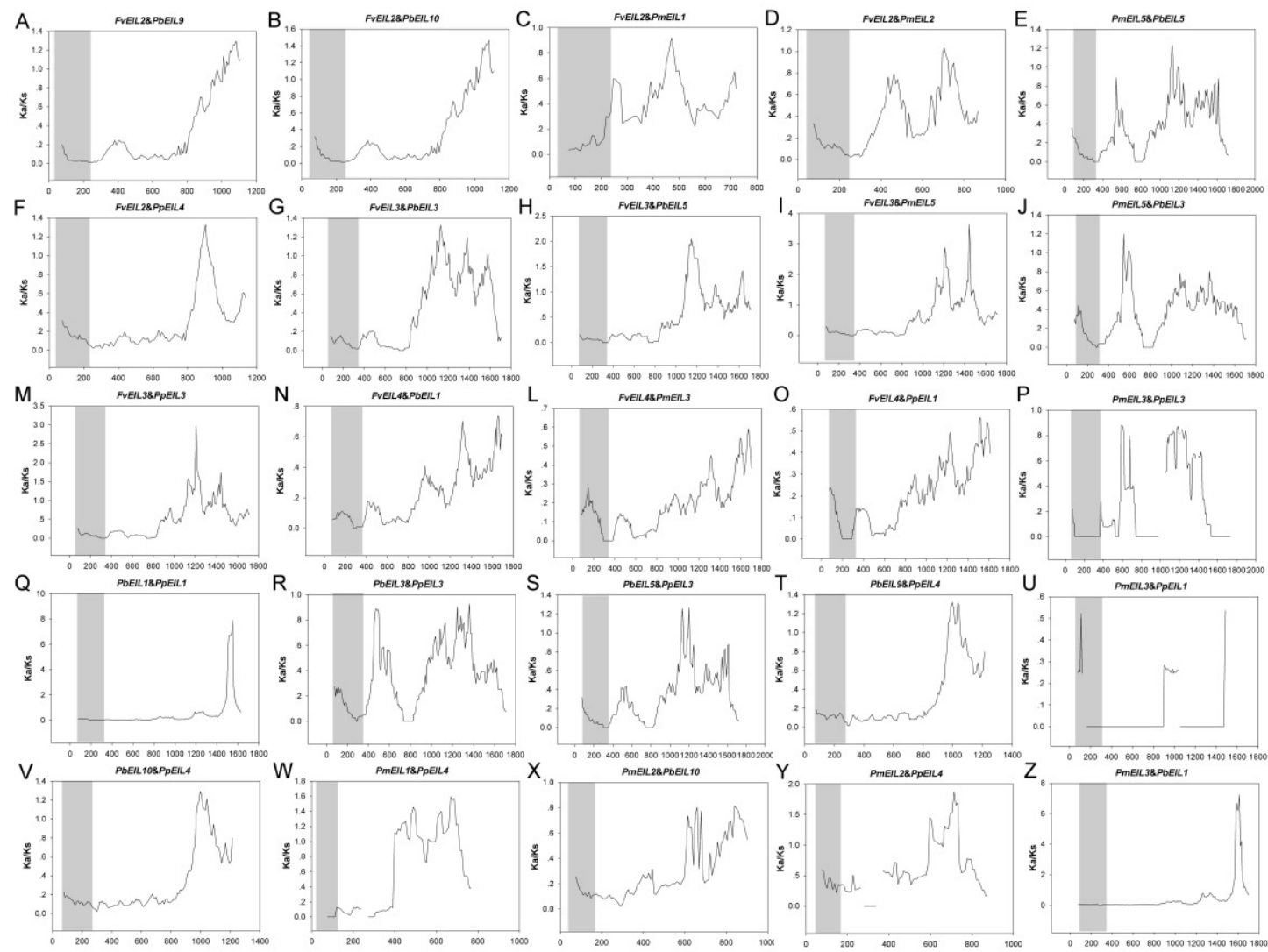


\section{Figure 7 (on next page)}

Figure 7. Expression profiling of pear PbEIL genes in eight samples from root, stem, leaves and fruits in several development stage .

The expression profile data was obtained qRT-PCR experiment. Blue and red colors indicate low-expression and high-expression, respectively. 


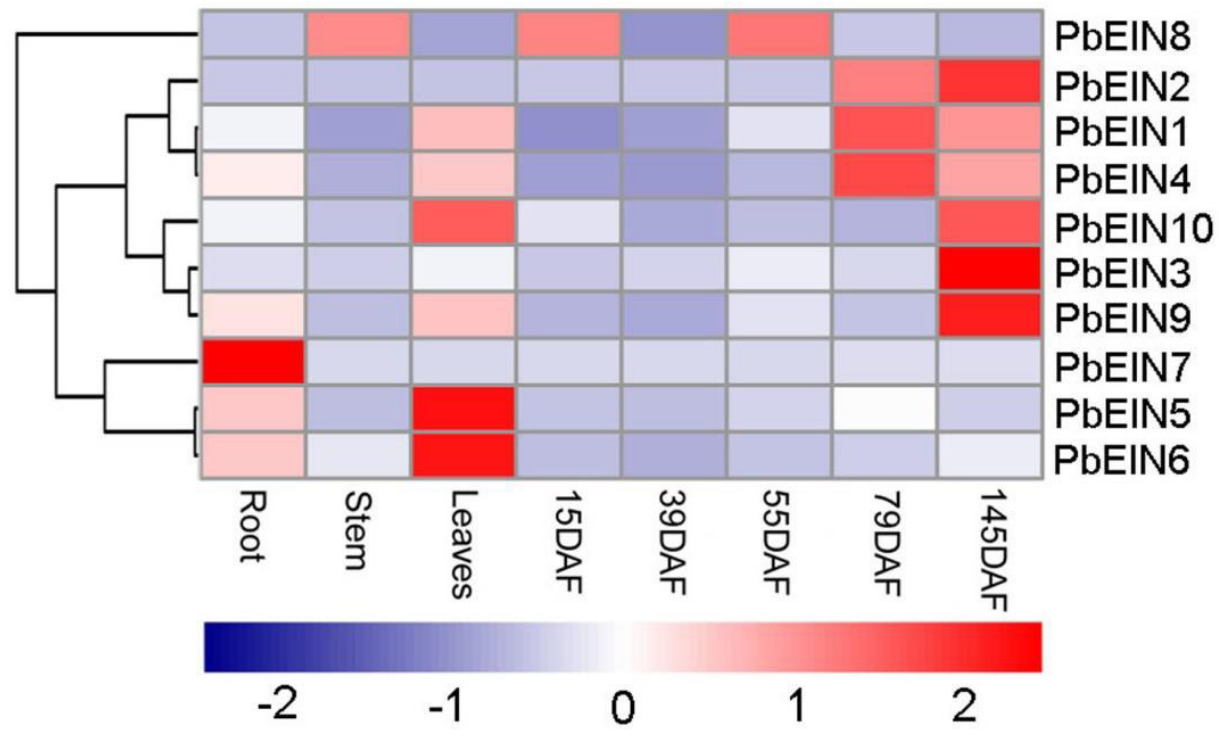




\section{Table $\mathbf{1}$ (on next page)}

Table 1. List of EIN3/EIL genes identified in pear, peach, mei and strawberry.

Note: Pear gene models are found in the GigaDB Genome database; mei and peach gene models are found in the Rosaceae Genome Database; strawberry gene models are found in the Phytozome database. 
1 Table 1. List of EIN3/EIL genes identified in pear, peach, mei and strawberry.

\begin{tabular}{|c|c|c|c|c|}
\hline Name & Gene Model & Chromosme & 5' End & 3' End \\
\hline FvEIL1 & mrna25474.1 & Chr1 & 17653671 & 17655527 \\
\hline FvEIL2 & mrna16361.1 & Chr1 & 18891616 & 18892965 \\
\hline FvEIL3 & mrna20650.1 & Chr3 & 29248944 & 29253704 \\
\hline FvEIL4 & mrna00379.1 & Chr7 & 290967 & 292781 \\
\hline FvEIL5 & mrna00392.1 & Chr7 & 349495 & 351202 \\
\hline PmEIL1 & Pm001950 & Chr1 & 15239829 & 15241073 \\
\hline PmEIL2 & Pm002057 & Chr1 & 16248534 & 16250294 \\
\hline PmEIL 3 & Pm017009 & Chr5 & 6907428 & 6909233 \\
\hline PmEIL4 & Pm017011 & Chr5 & 6933006 & 6934874 \\
\hline PmEIL5 & Pm028171 & scaffold103 & 1235430 & 1246520 \\
\hline PpEIL1 & ppa003493m & Chr2 & 5516222 & 5518429 \\
\hline PpEIL2 & ppa003550m & Chr2 & 5549949 & 5552334 \\
\hline PpEIL3 & ppa003113m & Chr6 & 3882268 & 3885188 \\
\hline PpEIL4 & ppa016118m & Chr6 & 16979366 & 16982360 \\
\hline PbEIL1 & Pbr024739.1 & Chr2 & 8493409 & 8495211 \\
\hline PbEIL2 & Pbr024740.1 & Chr2 & 8506285 & 8508129 \\
\hline PbEIL3 & Pbr000646.1 & Chr3 & 18718500 & 18721454 \\
\hline PbEIL4 & Pbr026603.1 & Chr8 & 3598382 & 3602224 \\
\hline
\end{tabular}




\begin{tabular}{lllll}
\hline PbEIL5 & Pbr004535.1 & Chr11 & 22794386 & 22798326 \\
PbEIL6 & Pbr033210.1 & Chr15 & 31009957 & 31014042 \\
& & & & \\
PbEIL7 & Pbr010447.1 & scaffold170.2. & 239188 & 246113 \\
& & 1 & & \\
PbEIL8 & Pbr010448.1 & scaffold170.2. & 259361 & 262132 \\
& & & & \\
& & 1 & & \\
PbEIL9 & Pbr022557.1 & scaffold341.0 & 56672 & 57973 \\
& & & & \\
PbEIL10 & Pbr039294.1 & scaffold837.0 & 82840 & 84144 \\
\hline
\end{tabular}

2 Note: Pear gene models are found in the GigaDB Genome database; mei and peach gene models are found in

3 the Rosaceae Genome Database; strawberry gene models are found in the Phytozome database. 


\section{Table 2 (on next page)}

Table 2. The relative syntenic quality of EIN3/EIL genes in four Rosaceae plants.

Note: the relative syntenic quality was estimated as twice the number of matches divided by the sum of the total number of genes in both conserved gene regions, based on the previous methods ( Cannon et al. 2003 ; Cannon et al. 2006 ) . 
1 Table 2. The relative syntenic quality of EIN3/EIL genes in four Rosaceae plants.

\begin{tabular}{|c|c|c|c|c|c|}
\hline & Clade A1 & Clade A2 & Clade B1 & Clade B2 & Average \\
\hline $\mathrm{Pb}-\mathrm{Pp}$ & & & $22.50 \%$ & $44.07 \%$ & $33.29 \%$ \\
\hline $\mathrm{Pb}-\mathrm{Pm}$ & & & $21.51 \%$ & $41.18 \%$ & $31.35 \%$ \\
\hline $\mathrm{Pb}-\mathrm{Fv}$ & & & $24.07 \%$ & $28.26 \%$ & $26.17 \%$ \\
\hline Pp-Pm & $26.67 \%$ & & $40.00 \%$ & $29.73 \%$ & $32.13 \%$ \\
\hline $\mathrm{Pp}-\mathrm{Fv}$ & $10.26 \%$ & & & & $10.26 \%$ \\
\hline \multirow[t]{2}{*}{$\mathrm{Pm}-\mathrm{Fv}$} & $10.00 \%$ & & $26.32 \%$ & $3.77 \%$ & $13.36 \%$ \\
\hline & & & & & $24.43 \%$ \\
\hline
\end{tabular}

2 Note: the relative syntenic quality was estimated as twice the number of matches divided by the sum of the

3 total number of genes in both conserved gene regions, based on the previous methods (Cannon et al. 2003;

4 Cannon et al. 2006).

5

\section{References}

7 Cannon SB, Mccombie WR, Sato S, Tabata S, Denny R, Palmer L, Katari M, Young ND, and Stacey G. 2003. Evolution and microsynteny of the apyrase gene family in three legume genomes. Molecular Genetics and Genomics 270:347-361.

Cannon SB, Sterck L, Rombauts S, Sato S, Cheung F, Gouzy J, Wang X, Mudge J, Vasdewani J, and Schiex T. 2006. Legume genome evolution viewed through the Medicago truncatula and Lotus japonicus genomes. Proceedings of the National Academy of Sciences of the United States of America 103:14959-14964. 